\title{
HyperspeCtRal ReflectanCE AND FluORESCEnCE IMAGing SyStem FOR FoOd QuALITY AND SAFETY
}

\author{
M. S. Kim, Y. R. Chen, P. M. Mehl
}

\begin{abstract}
This article presents a laboratory-based hyperspectral imaging system designed and developed by the Instrumentation and Sensing Laboratory, U.S. Department of Agriculture, Beltsville, Maryland. The spectral range is from 430 to $930 \mathrm{~nm}$ with spectral resolution of approximately $10 \mathrm{~nm}$ (full width at half maximum) and spatial resolution better than $1 \mathrm{~mm}$. Our system is capable of reflectance and fluorescence measurements with the use of dual illumination sources where fluorescence emissions are measured with ultraviolet $(U V-A)$ excitation. We present the calibrations and image-correction procedures for the system artifacts and heterogeneous responses caused by the optics, sensor, and lighting conditions throughout the spectrum region for reflectance and fluorescence. The results of the fluorescence correction method showed that the system responses throughout the spectrum region were normalized to within $0.5 \%$ error. The versatility of the hyperspectral imaging system was demonstrated with sample fluorescence and reflectance images of a normal apple and an apple with fungal contamination and bruised spots. The primary use of the imaging system in our laboratory is to conduct food safety and quality research. However, we envision that this unique system can be used in a number of scientific applications.
\end{abstract}

Keywords. Apples, Contamination, Disease, Defect, Bruise, Hyperspectral imaging, Reflectance, Fluorescence, Calibration.

$\mathrm{G}$ overnment agencies and industries have committed cooperative efforts to improve the overall quality and safety of foods produced in the United States. Rapid, noninvasive methods that can be implemented to assess hazardous conditions in food production would be substantially beneficial. Spectroscopic sensing techniques may allow on-line measurements with such specificity. Researchers at the Instrumentation and Sensing Laboratory (ISL), United States Department of Agriculture (USDA), Beltsville, Maryland, have been actively involved in the development of on-line detection systems. Approaches including machine vision, multispectral imaging, and spectral systems for poultry inspection are among those reported recently to differentiate wholesome vs. unwholesome chicken carcasses (Chen, 1993; Chen et al., 1998; Park et al., 1998). These systems have shown tremendous potential for on-line implementations. Others have also shown feasible use of

Article was submitted for review in August 2000; approved for publication by the Information \& Electrical Technologies Division of ASAE in March 2001.

Company and product names are used for clarity and do not imply any endorsement by USDA to the exclusion of other comparable products.

The authors are Moon S. Kim, Biophysicist, Yud-Ren Chen, Research Leader, and Patrick M. Mehl, Research Associate, Instrumentation and Sensing Laboratory, U.S. Dept. of Agriculture, Beltsville, Maryland. Corresponding author: M. S. Kim, USDA/ARS/ISL, Bldg. 303 BARC-East, 10300 Baltimore Ave., Beltsville, MD 20705-2350; phone: 301-504-8450; fax: 301-504-9466; e-mail: kimm@ba.ars.usda.gov. spectroscopic and imaging techniques to detect defects in apples and other agricultural products (Rehkugler and Throop, 1986; Pen et al., 1987; Upchurch et al., 1990; Miller et al., 1998; Tao and Wen, 1999).

Spectroscopic assessments with relatively small pointsource measurements have disadvantages compared to an imaging approach that characterizes the spatial variability of sample materials (Kim et al., 2001). In particular, imaging techniques are better suited for the detection of localized effects of a sample material. Lu and Chen (1998) pointed out that on-line imaging applications may only require several essential spectral bands (multispectral) to extract unwholesome conditions present in food commodities. However, the optimal spectral bands may depend on the commodity of interest and a number of other factors, such as the type of contamination and the state of the commodity. In order to define optimal spectral bands and further develop spectral algorithms for on-line implementation, more research is needed when considering the myriad of food commodities available. These factors point to a need for a detection system capable of both imaging and hyperspectral capabilities.

Hyperspectral imaging techniques have been already adapted in many scientific disciplines, from microscopic studies to airborne remote-sensing applications (Goetz et al., 1997; Martinsen et al., 1999; Borregaard et al., 2000). In general, these systems measure reflectance with spectral regions ranging from the visible (VIS) to short-wave infrared (SWIR) ranges of the solar spectrum. A volume of hyperspectral images consists of three-dimensional data that contain spatial information along with a high spectral resolution $(10 \mathrm{~nm})$ spectrum at each pixel location. 
In principle, two approaches to the acquisition of a cube of spatial and spectral data have been seen in the recent development of hyperspectral imaging techniques (fig. 1). One approach, illustrated in figure 1a, sequentially captures a full spatial scene at each spectral band to form a three-dimensional image cube. The use of multiple band-pass filters, a liquid-crystal tunable filter, or an acousto-optic tunable filter exemplifies this approach (Denes et al., 1997; Gat, 1998). Another approach (fig. 1b) is a pushbroom method in which a line of spatial information with a full spectral range per spatial pixel is captured sequentially to complete a volume of spatial-spectral data (Martinsen et al., 1999). Laboratory-based line-by-line hyperspectral imaging is feasible because of advancements in two-dimensional matrix detectors (i.e., CCD) and readily available holographic notch filters.

Fluorescence is a phenomenon in which light absorption at a given wavelength by a chromophore is followed by the emission of light, usually, at longer wavelengths (Stoke's shift). A number of compounds emit fluorescence in the VIS region of the spectrum when excited with ultraviolet (UV) radiation (Chappelle et al., 1991; Lang et al., 1992). Steady-state fluorescence techniques are generally regarded as sensitive optical tools and have proven to be useful for a number of scientific areas including cell biology and medical, forensic, and environmental sciences (Harris and Hartly, 1976; Chappelle et al., 1984; Albers et al., 1995). Fluorescence emission characteristics of food commodities can be changed by many factors. Exogenous contaminants, such as fecal and epiphytic pathogens, as well as intrinsic changes in food products resulting from contamination may contribute to changes in the fluorescence. However, the use of fluorescence measurements to this date has not been fully explored for the safety and quality assessment (on-line) of food commodities.

Under these criteria, we developed a laboratory-based hyperspectral imaging system capable of both reflectance and fluorescence measurements. The objectives of this

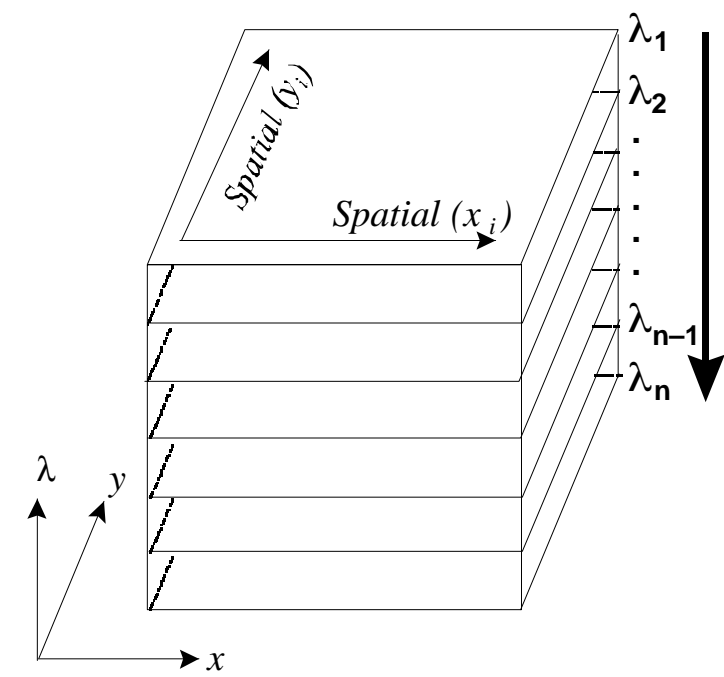

(a) methodology article are to provide a detailed description of the ISL hyperspectral imaging system, including its calibration and image-correction procedures, and to illustrate the versatility of the system with preliminary images from our ongoing apple study.

\section{HyPerspectral Imaging System SYSTEM DESIGN}

The ISL hyperspectral imaging system uses the line-by-line scanning technique accommodating a range of sample sizes. A modular design groups essential components together, providing easy access for replacement/upgrade of parts including $\mathrm{CCD}$, optics, and lighting peripherals. The system's structural frame $(30 \times 50 \times 75 \mathrm{~cm})$ was constructed with anodized aluminum columns enclosing all sensitive components and providing a rigid platform.

The imaging system was designed to cover VIS to near-infrared (NIR) regions of the spectrum for reflectance measurements. For fluorescence emissions with UV-A excitation, the blue to NIR regions, up to approximately $730 \mathrm{~nm}$ (chlorophyll $a$ fluorescence emission band), were essential for our applications. In addition, the imaging resolution of $1 \mathrm{~mm}$ was considered adequate to distinguish features of small anomalies we may encounter in many food products.

\section{HaRdWare Components}

Figure 2 shows the schematic diagram and hardware components of the ISL hyperspectral imaging system. The sensor module includes a back-illuminated CCD camera and a control unit (PixelVision, Inc., Tigard, Oregon). The CCD has $512 \times 512$-pixel elements and is thermo-electrically cooled by a two-stage Peltier device. The dynamic data range of the camera is 16-bit, and the raw pixel intensities are digitized and saved to a PC image file consisting of 16-bit unsigned integers.

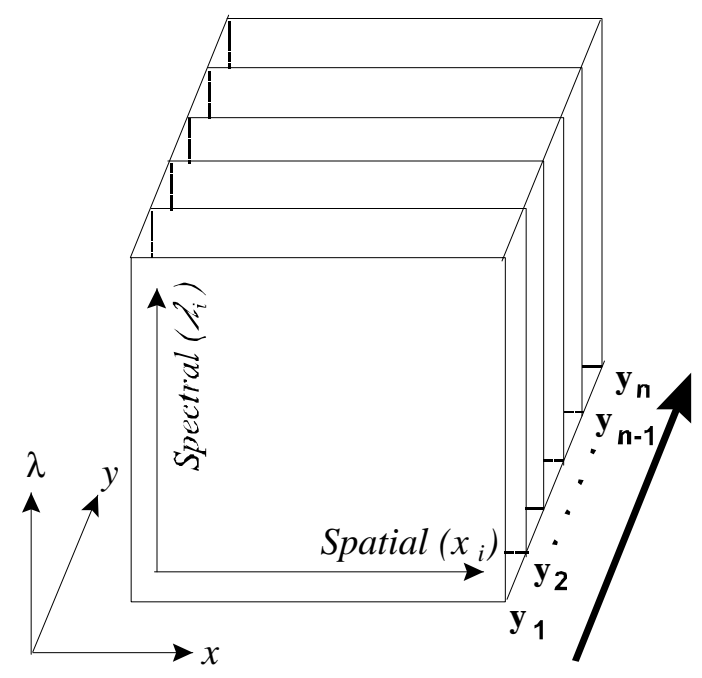

(b)

Figure 1. Conceptual representation of a volume of hyperspectral image data. Dark arrows indicate directions for sequential acquisitions to complete the volume of spatial and spectral data. 


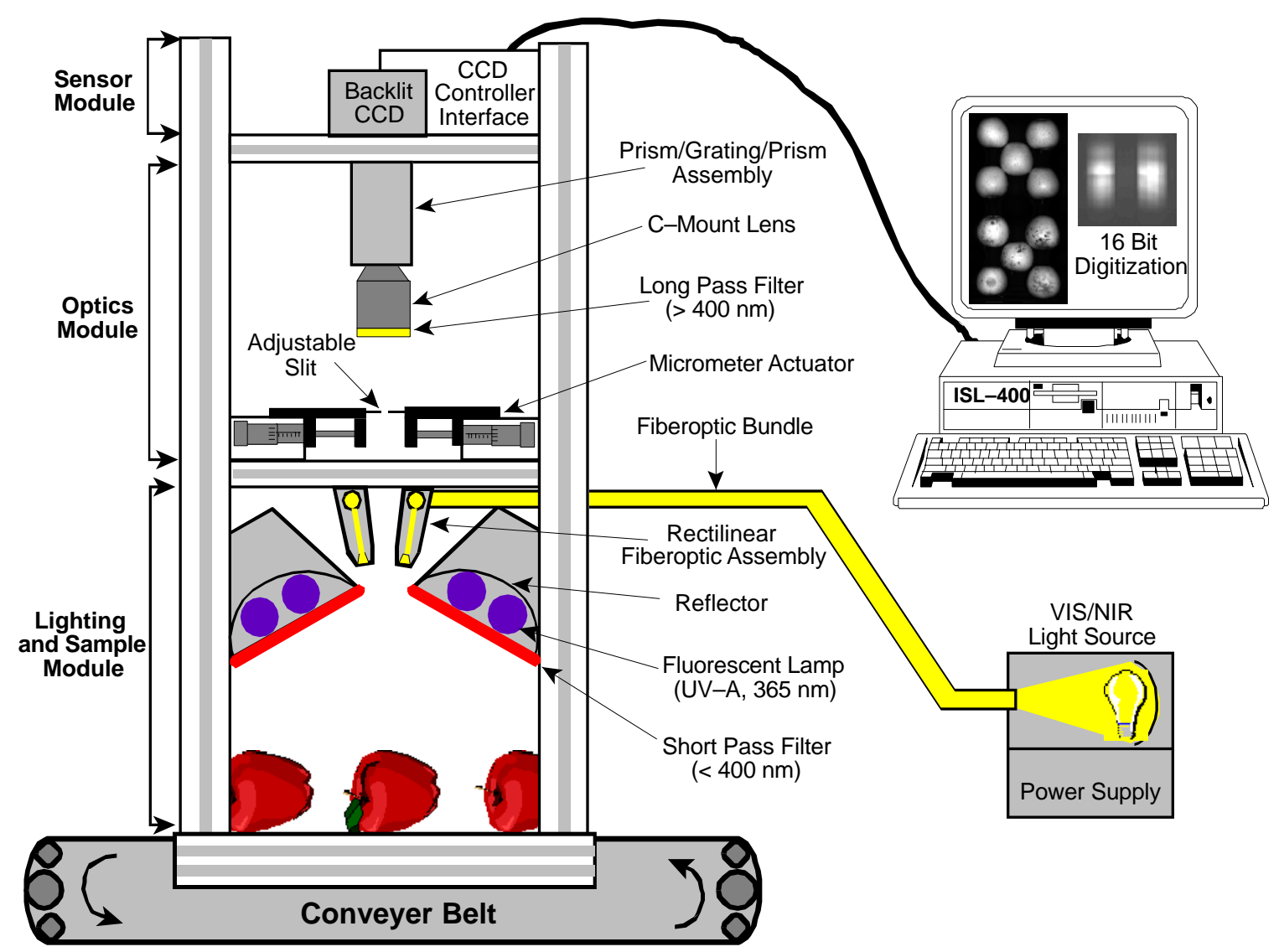

Figure 2. Schematic diagram and hardware components of ISL hyperspectral imaging system.

The optics module contains a spectrograph (ImSpectorV9, Spectral Imaging Ltd., Oulu, Finland) coupled with a standard f 1.4 C-mount lens (Schneider Optics Inc., Hauppauge, New York) attached to the CCD camera head. The ImSpector consists of prism-grating-prism (PGP) construction, which is a holographic transmission grating. The assembly disperses the incoming line of radiation into the spectral and spatial matrices and then projects them onto the CCD. The horizontal and vertical pixels on the CCD capture spatial and spectral information, respectively. A typical image contains 408 spatial pixels and 128 or 256 spectral bands, depending on the amount of spectral pixel binning chosen.

Along the optical path, an adjustable external slit made of two $10 \times 1 \mathrm{~cm}$ razor-edge metal blades, each mounted on a micrometer actuator and facing each other, is incorporated. This external slit attachment adjusts the width of the instantaneous field of view (IFOV), allowing modification of spatial resolution without adjusting the collecting lens aperture. This approach circumvents the deterioration of data quality, the chromatic and geometric aberrations, and the loss of system throughput caused when a small collecting lens aperture is used.

The lighting module uses two independent light sources to provide illumination for fluorescence and reflectance measurements. For reflectance measurements, two $150 \mathrm{~W}$ halogen lamps powered by regulated DC power supplies allow the light intensity to be adjusted. Light is transmitted through two randomly arranged rectilinear fiber bundles (Fiber-Lite A-240P, Dolan-Jenner Industries, Inc., Lawrence, Massachusetts) providing near-uniform illumination.
These line lights are mounted to illuminate the IFOV at $5^{\circ}$ forward and backward angles to minimize shadows and directional scattering caused by samples with 3-dimensional features.

For fluorescence measurements, two UV-A fluorescent lamp assemblies (Model XX-15A $365 \mathrm{~nm}$, Spectronics Corp., Westbury, New York) are arranged toward the IFOV at $30^{\circ}$ off-nadir, backward and forward, for near-uniform excitation energy to the sample area. Short-pass filters (UG1, Schott Glass Technologies Co., Duryea, Pennsylvania) are placed in front of the lamp housings to prevent transmittance of radiations greater than approximately $400 \mathrm{~nm}$ and thus eliminate spectral contamination by pseudo-fluorescence.

Food commodities normally move along a predetermined assembly or production line. A conveyor belt is used to transport sample materials through the line of IFOV in transverse direction while the stationary imaging system acquires data. The conveyor belt is $45 \mathrm{~cm}$ wide and equipped with an adjustable speed control (Speedmaster, Leeson Electric Corp., Grafton, Wisconsin), which allows line-byline sample acquisition with adjustable scanning rates $(0.1-5 \mathrm{~cm} / \mathrm{sec})$.

\section{OPERATION}

The imaging system is a laboratory-based system designed to be operated in a darkened room. Sample materials are placed on a tray painted with non-fluorescent, flat black paint to minimize background scattering. To complete a set of reflectance and fluorescence image data, a typical operation sequence consists of acquisitions of 1) dark 
current measurements, 2) reflectance of samples followed by the reference target, 3) fluorescence, and 4) a second set of dark current measurements. Note that a set of flat-field measurements (described later) to correct fluorescence images was acquired prior to the above measurements.

The speed of the conveyer belt was adjusted based on the predetermined CCD exposure time and data transfer rate to achieve approximately equivalent image size and spatial resolution for reflectance and fluorescence measurements. A typical fluorescence measurement took approximately twice as long ( $2 \mathrm{~s} /$ frame) as a reflectance measurement. Dark current measurements were acquired with the light source turned off and with the lens cap on. For spectral calibrations, images were acquired with no spectral binning (256 spectral bands), and two-pixel spectral binning was used for the apple measurements presented in this article.

\section{SOFTWARE}

Adjustment of the system parameters and the data acquisition were controlled by Microsoft Windows 98-based interface software (PixelView V3.1) provided by the CCD camera manufacturer. We developed processing software in MS Visual Basic, operating in the MS Windows 98 environment, to convert the raw data files to a volume of monochromatic band image data. In addition, dark current subtraction, image corrections for reflectance and fluorescence measurements (methods described later), image visualization/enhancement, simple arithmetic operations, spectral and spatial data retrieval, and other functions can be performed with this software. It also allows us to save an individual spectral band image as a standard bitmap for presentation purposes or a volume of data in the file format required by commercial packages such as ENVI (Environment for Visualizing Images, Research Systems, Inc., Boulder, Colorado) for further analysis.

\section{System Calibrations and Image Corrections}

Calibrations and data corrections for a hyperspectral imaging system are complex due to a multitude of spectral bands, usually numbering over one hundred, along with associated spatial domains. The reflectance and fluorescence capabilities incorporated in our system augment the complexity. Instrument artifacts - such as heterogeneity in CCD responses, illumination, and optics - degrade the performance and data quality. These heterogeneities throughout the wavelength region can be corrected by using flat-field (homogeneous) reference measurements illuminated by the light source of the system. However, different approaches were necessary for reflectance and fluorescence measurements, as illustrated later.

Calibration and image-correction procedures conducted in our laboratory included both recommendations by the PGP manufacturer and independent approaches implemented by the investigators. Optical parts along the light path of the system were tested with a spectrofluorometer (Spex, JY Inc., Edison, New Jersey) to ensure that they were non-fluorescent.

\section{Spectral and Spatial Calibrations}

Spectral calibrations were performed using $\mathrm{Hg}-\mathrm{Ne}, \mathrm{Ne}$, $\mathrm{Ar}$, and $\mathrm{Kr}$ pencil lights (Oriel Instruments, Stratford, Connecticut). A linear regression method with the spectral emission peak wavelengths of the lamps vs. corresponding channel number of the imaging system was used to obtain wavelength values for individual channels. The spectral range of the system was found to be 428 to $930 \mathrm{~nm}$. In addition, the full width at half maximum (FWHM) of the system was determined to be approximately $10 \mathrm{~nm}$ throughout the spectral range. Note that rotation of the PGP spectrograph was aligned with respect to the vertical and horizontal axes of the CCD prior to the spectral calibration.

A two-inch square standard grid (US Air Force 1951 plate, Edmund Scientific Co., Barrington, New Jersey) was used to determine spatial resolution of the system. With the addition of the adjustable slit, we were able to optimize light throughput and achieve $1 \mathrm{~mm}$ spatial resolution. A detailed description of spectral and spatial calibration procedures is omitted for brevity, and readers are referred to articles by $\mathrm{Lu}$ and Chen (1998) and Mehl et al. (2000).

\section{CCD RESPONSIVITY}

Responsivity of a photon detector can be evaluated as a function of changes in detector responses with respect to integration (exposure) times under a fixed light intensity. Another approach is to evaluate the detector responses with changing light intensities under a fixed integration time. Either method would suffice in evaluating responsivity of a photon detector (note that this may not be true for biological systems). We used the first method to evaluate linear responsivity of the $\mathrm{CCD}$ in the hyperspectral imaging system.

The evaluation was performed at four wavebands $(450$, $550,650$, and $800 \mathrm{~nm})$ and four pixel locations $(50,150,250$, and 350) along the spatial axis per band. The fiber optic line lights, in conjunction with a white Spectralon panel (Labsphere Inc., North Sutton, New Hampshire), provided a diffused flat-field (homogeneous) target. Images were acquired with a range of CCD exposure times, for instance: $50 \mathrm{~ms}, 100 \mathrm{~ms}$, and 200 to $2200 \mathrm{~ms}$ by $200 \mathrm{~ms}$ increments for $550 \mathrm{~nm}$. The minimum and maximum exposure times were selected to span nearly the full dynamic range of the CCD (A/D converter).

Figure 3 show a graph of pixel intensity at $550 \mathrm{~nm}$ plotted against exposure time for pixel location 150. Each point on the graph represents an average pixel intensity of 10 measurements (dark current subtracted) of the target. The results clearly demonstrate a linear relationship $\left(r^{2}=.9999\right)$ between the exposure time and CCD response throughout the dynamic data range. Similar results $\left(r^{2}>.999\right)$ were obtained for the other three locations along the spatial axis at $550 \mathrm{~nm}$ and for the 450, 650, and 800 bands at all four locations (data not shown).

\section{FLAT-FIELd CORRECTIONS Reflectance}

A uniform photometric response throughout the CCD matrix can be achieved by calculating reflectance factors $(\mathrm{RF})$, which are the ratios of reflected radiation from a sample to those of a known reference material. An RF value for an individual pixel (i) at a given wavelength $(\lambda)$ was calculated using following equation: 


$$
\mathrm{RF}_{\mathrm{i} \lambda}=\frac{\mathrm{RS}_{\mathrm{i} \lambda}-\mathrm{RD}_{\mathrm{i} \lambda}}{\mathrm{RR}_{\mathrm{i} \lambda}-\mathrm{RD}_{\mathrm{i} \lambda}} \times \mathrm{RC}_{\lambda}
$$

where

RS = sample image

$\mathrm{RD}=$ dark current image

$\mathrm{RR}=$ reference image

$\mathrm{RC}=$ correction factor for the reference panel.

The white Spectralon panel has a correction factor of .99 in the spectral region covered by the hyperspectral imaging system. However, RC $=1.0$ was used in our applications for simplification. The exposure times of the CCD and the light intensities were adjusted so that the maximum intensity levels of the reference were approximately one tenth less than that of the full dynamic range. Typically, averages of 20 reference and 20 dark current images were used in our applications. RF values have a range of 0.0 to 1.0 . Prior to saving the corrected images to PC, RF values were multiplied by 10000 to better utilize the dynamic range of the 16-bit integer and to reduce rounding errors.

\section{Fluorescence}

Because of limited spectral ranges in fluorescence emission $(<150 \mathrm{~nm})$, several flat-field materials were required to perform fluorescence flat-field corrections for the spectrum region of interest $(430-730 \mathrm{~nm})$. Fluorescein and rhodamine B (Sigma, St. Louis, Missouri) in methanol $(0.5 \mathrm{~g} / \mathrm{l}$ for both) were chosen as flat-field materials for the green and red region of the spectrum, respectively. A turbid fluorescent solvent is ideal for flat-field correction. However, for the correction in the blue region of the spectrum, after evaluations of numerous materials, a premium white inkjet paper (Union Camp Co.) exhibiting uniform blue fluorescence was used. This was an inexpensive alternative for a costly blue fluorophore for a routine calibration. The emission characteristics of these flat-field materials acquired with the Spex spectrofluorometer are shown in figure $4 \mathrm{a}$. The spectra were acquired with $365 \mathrm{~nm}$ excitation, $2 \mathrm{~nm}$ excitation and emission slit widths, at $2.5 \mathrm{~nm}$ spectral interval.

A total of 20 correction images for each flat-field material was acquired with the hyperspectral imaging system. For fluorescein and rhodamine $\mathrm{B}$, each solution was poured $1.5 \mathrm{~cm}$ deep in a non-fluorescent flat tray that was larger than

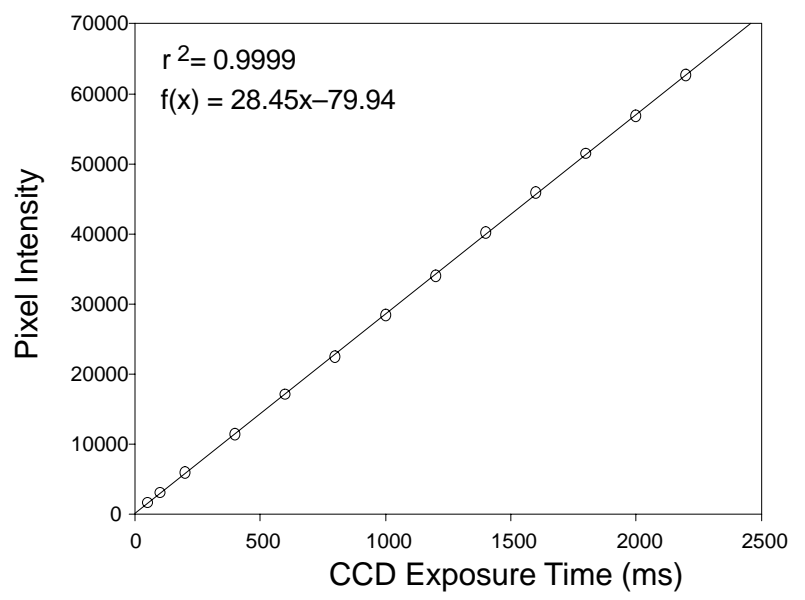

Figure 3. Relationship between CCD exposure time and response at $550 \mathrm{~nm}$. the IFOV. The CCD exposure time was adjusted so that the peak intensity for each material was slightly less than one-half of the full dynamic data range.

Figure $4 \mathrm{~b}$ shows emission spectra of the flat-field materials acquired with the hyperspectral imaging system. Each spectrum represents an average of 20 measurements of a randomly selected pixel. The spectra show slight variations in spectral shapes compared to those shown in figure 4a. These can be attributed to differences in spectral responses due to the broader excitation source, spectral bandwidth, and quantum efficiency of the imaging system. The three different shades of gray under the spectra shown in figure $4 \mathrm{~b}$ represent the part of spectrum used for the flat-field corrections. The spectrum regions for the white paper, fluorescein, and rhodamine B were approximately 430 $510 \mathrm{~nm}, 511-575 \mathrm{~nm}$, and $576-735 \mathrm{~nm}$, respectively.

Graphs shown on the left in figure 5 show uncorrected flat-field intensities along the spatial axis of the CCD at

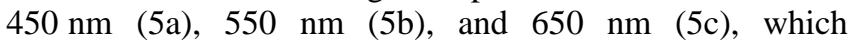
correspond to the intensities of white paper, fluorescein, and rhodamine $\mathrm{B}$, respectively. The parabolic curvatures of the uncorrected plots across the spatial axes reflect artifacts of optics in the system, because of which throughput toward the center was greater than at the edges. Peaks and valleys observed along these curvatures and variations seen at the
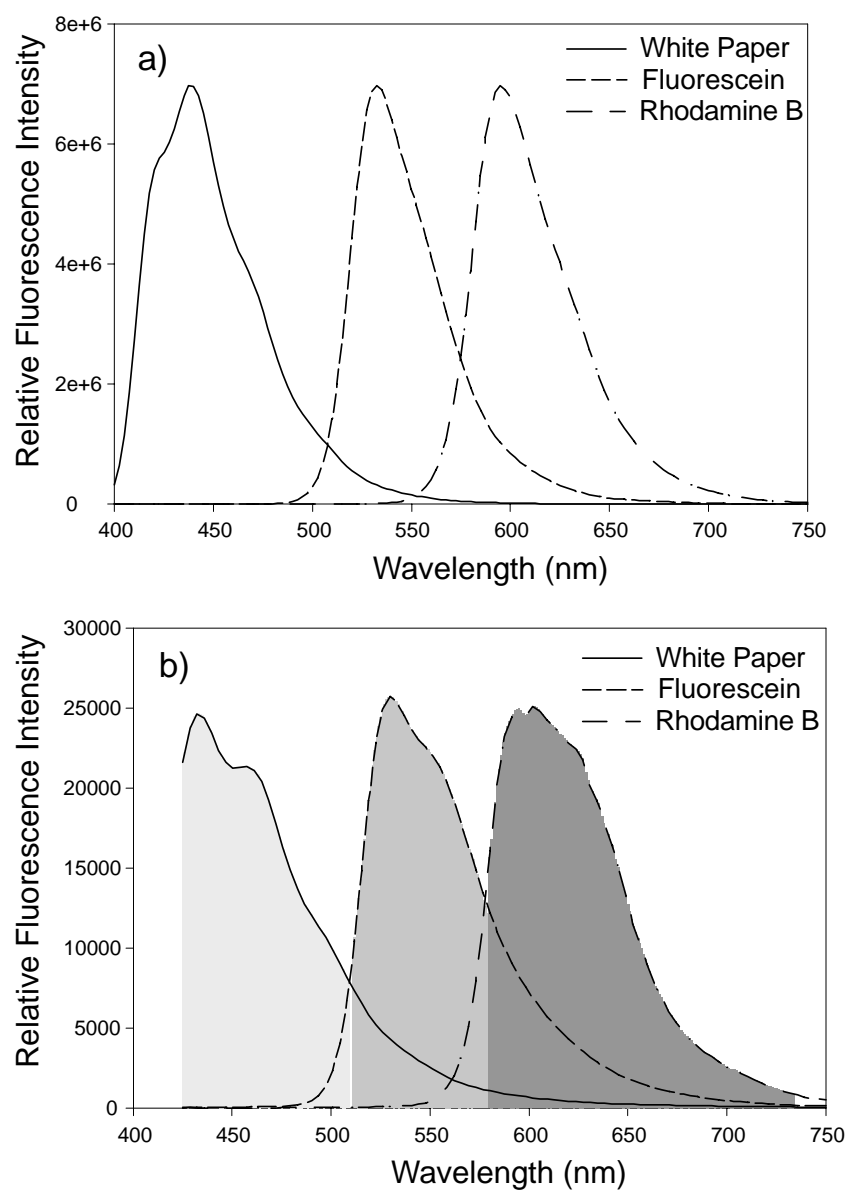

Figure 4. Fluorescence emission characteristics of flat-field targets (white paper, fluorescein, and rhodamine B). Emission spectra acquired with (a) a spectrofluorometer and (b) the ISL-hyperspectral imaging system using $365 \mathrm{~nm}$ excitation. 
same pixel regions between the three wavelengths are indicative of heterogeneous responses of the CCD matrix. Gray-scale images seen on the top portion of the graphs in figure 5 represent 20 measurements used to create the uncorrected plots. For each image, the gray scales were stretched to span the minimum and maximum pixel intensity values, which corresponded to black and white, respectively. The heterogeneous responses along the spatial pixels and between the spectral pixels are also well illustrated in these images. Histograms of uncorrected flat-field measurements are shown on the right in figure 5. These are plots of pixel intensities plotted against the pixel frequencies. These also illustrate a range and frequency of pixel intensity values for the uncorrected flat-field targets.

Fluorescence intensities are measures of quantum yields and are expressed in terms of relative fluorescence intensities (RFI). By nature, fluorescence emission is different from reflectance. The ratio method (i.e., RF) used in the

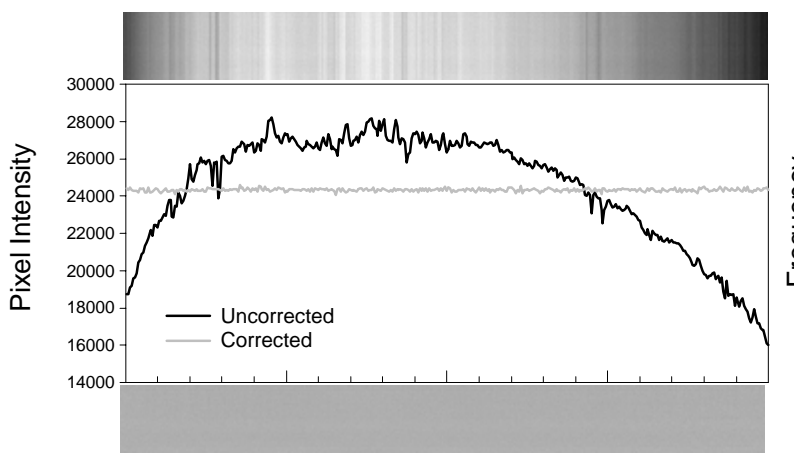

(a)
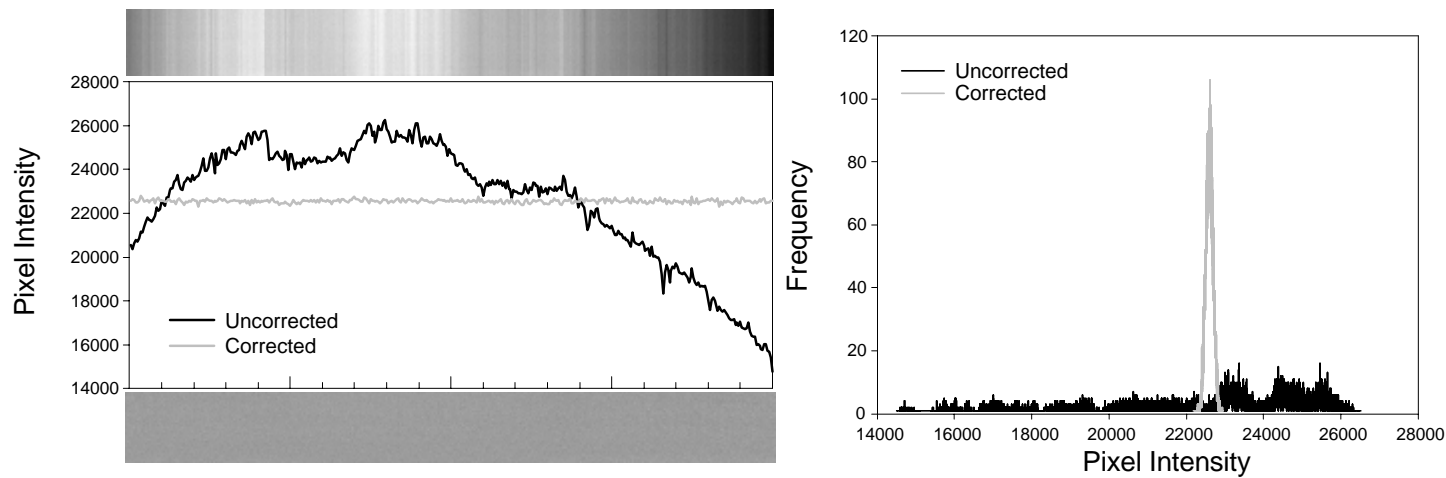

(b)
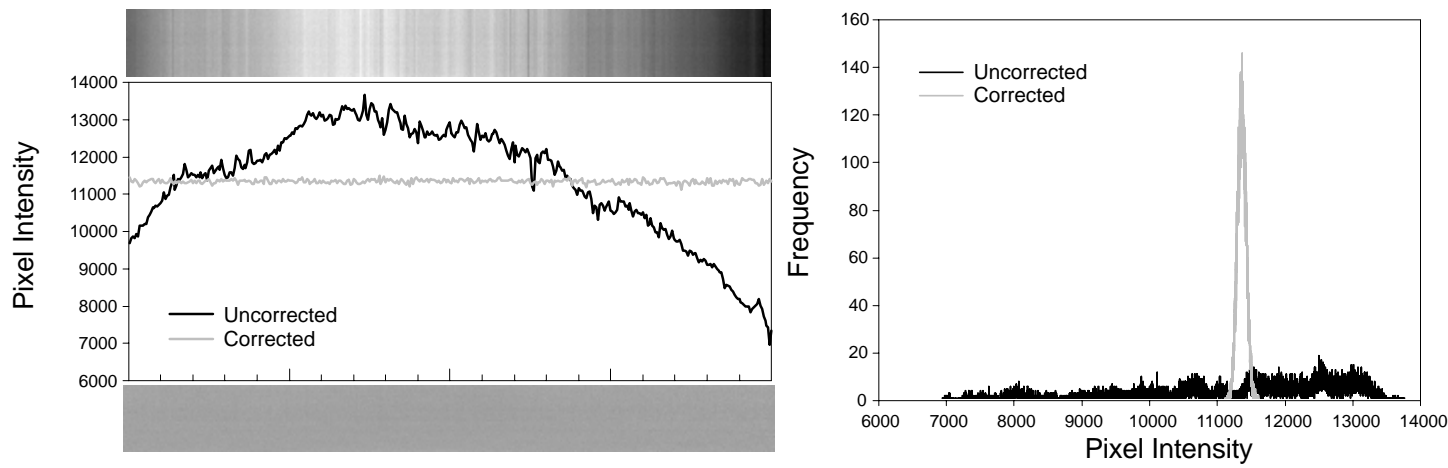

(c)

Figure 5. Spatial variations of CCD responses characterized with the flat-field target materials before and after the correction at: (a) $450 \mathrm{~nm}$, (b) 550 $\mathrm{nm}$, and (c) $650 \mathrm{~nm}$. Gray-scale images above and below each graph represent 20 measurements each, before and after the flat-field correction, respectively. Histograms on the right exhibit the pixel intensity plotted against pixel frequency of the gray-scale images before and after the correction. 
reflectance correction was not applicable for fluorescence correction. Instead of a ratio to a known reference material, individual pixel response obtained from a flat-field material is normalized to the average of all the pixel intensities for each wavelength. Hence, a correction factor (FC) for an individual CCD pixel (FF) was calculated as the fraction to the average of all pixel intensities $\left(\mathrm{FF}_{\mathrm{m}}\right)$ at that wavelength. As shown in the following equation, dark current (FD) subtraction was performed prior to performing the calculation of the fraction:

$$
\mathrm{FC}_{\mathrm{i} \lambda}=\frac{\mathrm{FF}_{\mathrm{m} \lambda}-\mathrm{FD}_{\mathrm{i} \lambda}}{\mathrm{FF}_{\mathrm{i} \lambda}-\mathrm{FD}_{\mathrm{i} \lambda}}
$$

To obtain a flat-field corrected sample (FCS), dark-current corrected sample image (FS) value was multiplied by FC as:

$$
\mathrm{FCS}_{\mathrm{i} \lambda}=\left(\mathrm{FS}_{\mathrm{i}}-\mathrm{FD}_{\mathrm{i} \lambda}\right) \times \mathrm{FC}_{\mathrm{i} \lambda}
$$

In order to obtain radiometrically corrected responses, an emission correction is required at each wavelength. Since individual pixel responses were normalized to the pixel mean $\left(\mathrm{FF}_{\mathrm{m}}\right)$ at a given wavelength, only a constant adjusting $\mathrm{FF}_{\mathrm{m}}$ for the radiometric correction was needed for all individual pixels. However, this step was omitted for simplicity since we were only interested in relative intensity changes in the sample materials. In addition, changes in the excitation light intensity over time affect the relative fluorescence intensity of a sample. However, the use of fluorescence band ratios may circumvent the variations caused by the changes in the excitation light intensity, and thus can provide a means for sample comparisons over time.

A new set of measurements, 20 each for white paper, fluorescein, and rhodamine B, were conducted to evaluate the correction method. The results of the flat-field corrections with the use of white paper at $450 \mathrm{~nm}$, fluorescein at $550 \mathrm{~nm}$, and rhodamine $B$ at $650 \mathrm{~nm}$ as sample materials are presented in figure $5 \mathrm{a}, 5 \mathrm{~b}$, and $5 \mathrm{c}$, respectively. Compared to the plots of uncorrected pixel responses, the flat-field corrections clearly resulted in uniform photometric responses throughout the spatial axes. The gray-scale images seen at the bottom portion of the graphs in figure 5 are the result of flat-field correction (20 measurements under the same gray-scale levels as the uncorrected images). The histogram plots in figure 5 show narrow pixel intensity ranges with normal distributions at 450, 550, and $650 \mathrm{~nm}$. Signal-to-noise ratios greater than 200 (equivalent to $0.5 \%$ error) were achieved at these wavelengths.

\section{Application to ApPles}

As a part of ongoing ISL food safety and quality research, over 2000 apples, including wholesome, contaminated, and defective samples, were collected from a Pennsylvania grower (Rice Fruit Co.) during the 1999 harvest season. Samples at the grower were randomly picked from either the trees or the producer's crates containing freshly harvested apples. In this article, hyperspectral images of two apples (variety 'Golden Delicious') selected from our image data sets are presented. The first sample was normal (healthy tissue) and the second had a fungal (sooty blotch) contamination along with a bruise spot (necrotic tissue), approximately $1 \mathrm{~cm}$ in diameter.
Gray-scale images of the apples, reflectance at 680 (R680) and $800 \mathrm{~nm}$ (R800), and fluorescence at 530 (F530) and $685 \mathrm{~nm}$ (F685), are shown in figure 6a, 6b, 6c, and 6d, respectively. Within each image, the apple on the left is the normal and the one on the right is fungal-contaminated with a bruise. The graph below each image represents pixel intensities across the dotted horizontal line in the image. In general, the reflectance images appear to exhibit more decrease in intensity toward the apple edges than the fluorescence images. This observation may suggest that target depth and directional factors may affect reflectance measurement more than fluorescence emission.

The images in figure 6 show good details, although clarity of spatial features appears to be dependent upon wavelength and measurement method. Lenticels on the apple surfaces are visible in the R680 image but not visible in the R800 image. In addition, the bruised spot is depicted in R680 but not in R800, while the fungal-contaminated spot is visible in both. Lenticels were not clear in the fluorescence images. However, F530 shows the contaminated spots and bruised spot with greater contrasts than the reflectance images.

The wavelengths for figure 6 were chosen after consideration of figure 7 , which contains reflectance (7a) and fluorescence (7b) spectra extracted from the hyperspectral image data. The pixel base of the spectra ranged from a single spatial point for sooty blotch to an average of 300 points for normal healthy tissue. The spectra shown in figure 7 were smoothed (three-point running average). Apple reflectance from 400 to $700 \mathrm{~nm}$ is predominantly affected by strong absorption characteristics of plant pigments, such as chlorophyll $a$ and $b$ and carotenoids (Abbott et al., 1997). In the healthy tissue spectra, the most predominant feature is the absorption characteristic of chlorophyll $a$ at around $680 \mathrm{~nm}$ (R680). Note that the healthy tissue spectrum (dotted line) in figure 7 was retrieved from a healthy region of the fungal-contaminated apple. R680 of healthy tissue from the fungal-contaminated apple is higher than that of the normal apple. The sooty blotch and bruised spot spectra are markedly different from the healthy tissue spectra, showing diminished characteristics from 500 to $680 \mathrm{~nm}$. Higher reflectance in NIR compared to VIS are seen due to the absence of light-absorbing compounds coupled with high scattering characteristics of fruit cells.

Figure $7 \mathrm{~b}$ shows higher fluorescence emissions from the healthy tissues compared to fungal-contaminated and bruised spots. Most plant materials, when illuminated with UV radiation, exhibit a broad-blue-green fluorescence emission with maxima at 450 and $530 \mathrm{~nm}$ (Chappelle et al., 1984). A multitude of plant constituents is responsible for fluorescence emission in the blue and green regions of the spectrum. Some of these include phenolic compound, riboflavin (FAD and FMN), NADPH, and carotenoid species (Chappelle et al., 1991; Lang et al., 1992). The mechanisms and plant constituents governing fluorescence emission in the blue-green region of the spectrum are complex and need further research. Fluorescence emissions in the 650-750 nm region are due to chlorophyll $a$ with emission maxima at $685 \mathrm{~nm}$ and $730 \mathrm{~nm}$ (Papageorgiou, 1975). The higher chlorophyll fluorescence emissions observed for the healthy tissues are the manifestation of higher concentrations compared to the bruised and contaminated spots (probably due to degradation of chlorophyll $a$ and attenuation of the excitation light, respectively). In addition, it is well known 


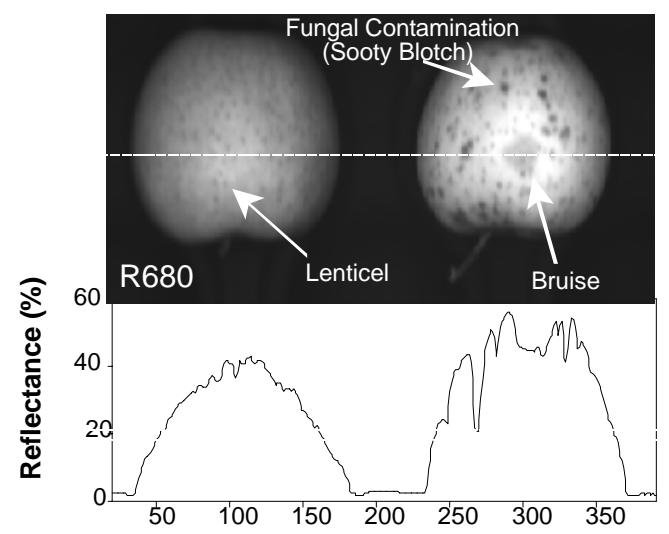

(a)

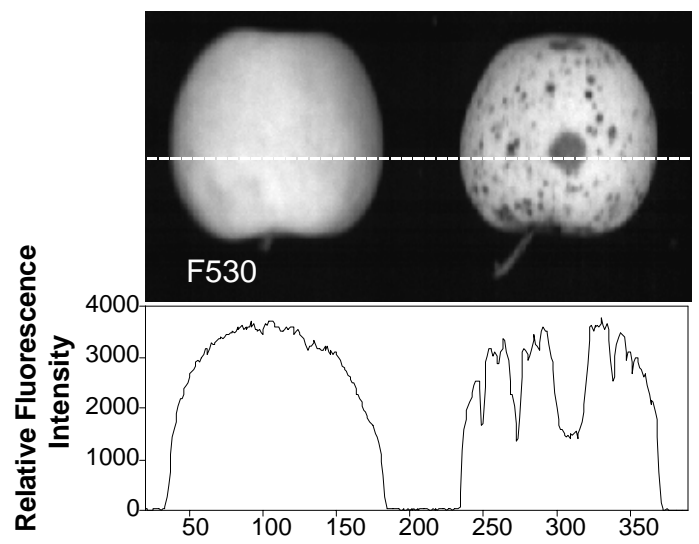

(c)

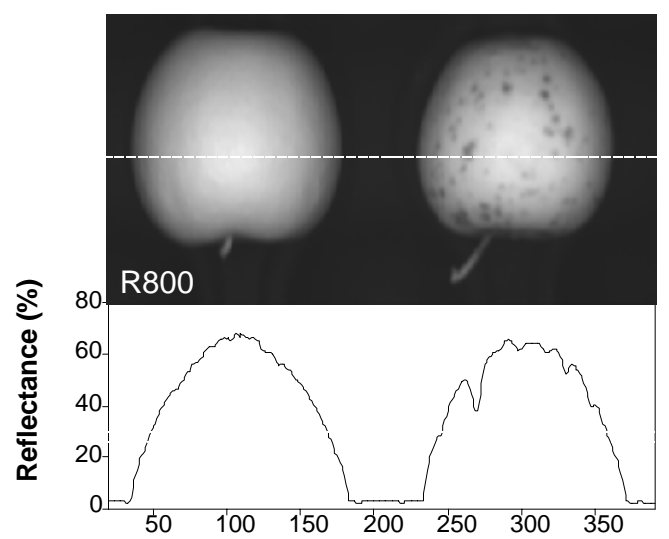

(b)

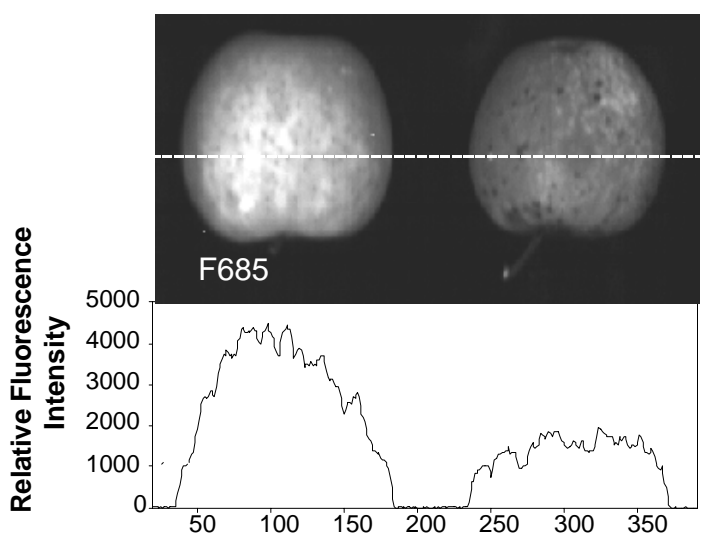

(d)

Figure 6. Examples of corrected images of apples, 'Golden Delicious' variety: (a) reflectance at $680 \mathrm{~nm}$, (b) reflectance at $800 \mathrm{~nm}$, (c) fluorescence at $530 \mathrm{~nm}$, and (d) fluorescence at $685 \mathrm{~nm}$. Pixel intensities along the dotted line across each image are presented in the graph below each image.

that chlorophyll a fluorescence (F685) is inversely proportional (photochemical quenching) to photosynthetic activity. These properties have been utilized by several investigators to assess fruit qualities (Smillie et al., 1987; DeEll et al., 1996).

Examination of the spectra provided a detailed elucidation of spectral features of apples and their variations caused by contamination and defect. This can enhance the interpretation of spatial variations in the images. For instance, an opposite trend in intensities for these apples between two chlorophyll band images, R680 and F685, are observed. These images indicate that the apple on the left in figure 6 contains a higher concentration of chlorophyll $a$. A greater relative intensity difference between the apples seen in F685 compared R680 (figs. 6a and 6d) demonstrates the sensitive nature of fluorescence techniques. More elaborate studies are being conducted by the authors using both reflectance and fluorescence imaging techniques for the detection of defects and contaminations in food commodities (e.g., poultry and apples).

\section{Conclusions}

We have designed and developed a laboratory-based hyperspectral imaging system with several unique features. The system is capable of capturing reflectance and fluorescence images in the 430 to $930 \mathrm{~nm}$ region with $1 \mathrm{~mm}$ spatial resolution. Fluorescence emission images were achieved with broad UV-A excitation, approximately 330 to $400 \mathrm{~nm}$ with a peak at $365 \mathrm{~nm}$. Image-correction procedures were presented to produce corrected images for reflectance and fluorescence methods. The evaluation of the flat-field correction method showed that heterogeneous responses of the system due to the optics, sensor, and lighting conditions throughout the spectrum region were normalized to within $0.5 \%$ error.

We envision that multispectral imaging, rather than hyperspectral imaging, will become an integral part of food production industries in the near future for automated on-line applications because of acquisition and real-time processing speeds. However, hyperspectral imaging systems are needed in order to define optimal bands and develop algorithms for many food commodities. For this purpose, we strongly believe that the ISL hyperspectral imaging system is a versatile platform, able to evaluate reflectance and fluorescence images at a high spectral resolution $(10 \mathrm{~nm})$.

Fluorescence emissions from food products are functions of complex biochemical and physicochemical properties. Further biochemical research is needed to identify compounds and mechanisms responsible for changes in fluorescence emissions. Nevertheless, as illustrated in this article with apples, the use of fluorescence techniques appears promising for detection of defects and contaminations. In addition, the complementary uses of both reflectance and fluorescence methods, to our knowledge, have never been explored for food safety and quality studies. 

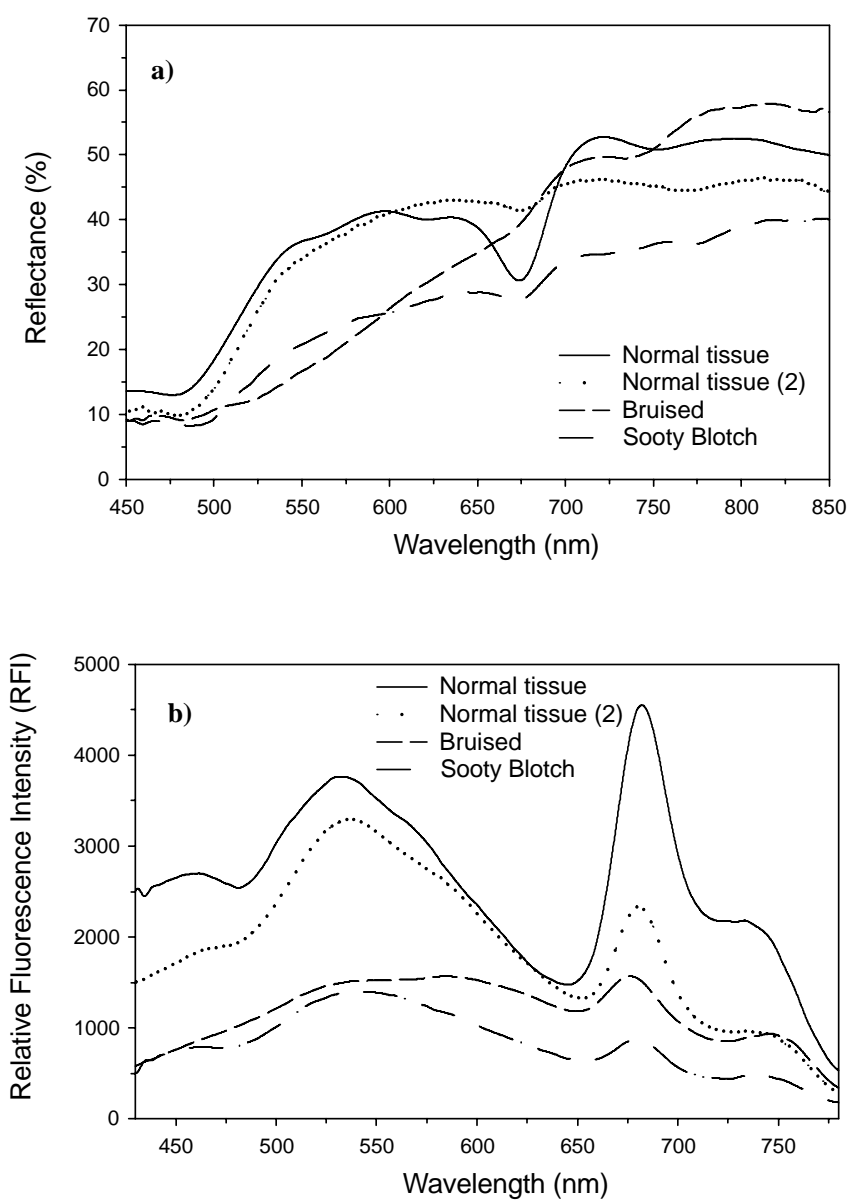

Figure 7. Reflectance (a) and fluorescence (b) spectra obtained from the volume of hyperspectral image data of apples.

With the use of the newly developed ISL hyperspectral imaging system, a novel approach using both techniques can be developed.

\section{ACKNOWLEDGMENTS}

The authors would like to thank the management at Rice Brother Fruits Company for providing access to their facilities. We would like to express our gratitude to Dr. William Hruschka and Ms. Diane Chan of the Instrumentation and Sensing Laboratory, USDA, for reviewing the manuscript and providing insightful comments.

\section{REFERENCES}

Abbott, J. A., R. Lu, B. L. Upchurch, and R. L. Stroshine. 1997. Technologies for non-destructive quality evaluation of fruits and vegetables. Horticultural Reviews 20: 1-120.

Albers, B., J. DiBenedetto, S. Lutz, and C. Purdy. 1995. More efficient environmental monitoring with laser-induced fluorescence imaging. Biophotonics Int. Nov.: 42-54.

Borregaard, T., H. Nielsen, L. Norgaard, and H. Have. 2000. Crop-weed discrimination by line imaging spectroscopy. $J$. Agri. Eng. Res. 75: 389-400.

Chappelle, E. W., F. M. Wood, J. E. McMurtrey, and W. W. Newcomb. 1984. Laser induced fluorescence of green plants: 1 A technique for the remote detection of plant stress and species differentiation. Appl. Optics 23: 134-138.
Chappelle, E.W., J. E. McMurtrey, and M. S. Kim. 1991. Identification of the pigment responsible for the blue fluorescence band in laser induced fluorescence (LIF) spectra of green plants, and the potential use of this band in remotely estimating rates of photosynthesis. Remote Sens. Environ. 36: 213-218.

Chen, Y. R. 1993. Classifying diseased poultry carcasses by visible and near-IR reflectance spectroscopy. Optics in Agriculture and Forestry. SPIE 1836: 46-55.

Chen, Y. R., B. Park, R. W. Huffman, and M. Nguyen. 1998. Classification of on-line poultry carcasses with backpropagation neural networks. J. Food. Proc. Eng. 21: 33-48.

DeEll, J. R., R. K. Prange, and D. P. Murr. 1996. Chlorophyll fluorescence of Delicious apples at harvest as a potential predictor of superficial scald development during storage. Postharvest Biol. Technol. 9(1): 1-6.

Denes, L. J., M. Gottlieb, and B. Kaminsky. 1997. Acousto-optic tunable filters in imaging applications. Opt Eng. 37: 1262-1267.

Gat, N. 1998. Real-time multi- and hyper-spectral imaging for remote sensing and machine vision: An overview. ASAE Paper No. 98-3027. St. Joseph, Mich.: ASAE.

Goetz, A. F. H., J. W. Boardman, B. Kindel, and K. B. Heidebrecht. 1997. Atmospheric corrections: On deriving surface reflectance from hyperspectral imagers. SPIE 3118: 14-22.

Harris, P. J., and R. D. Hartley. 1976. Detection of bound ferulic acid in cell walls of the Gramineae by ultraviolet fluorescence microscopy. Nature 259: 508-510.

Kim, M. S., J. E. McMurtrey, C. L. Mulchi, C. S. T. Daughtry, E. W. Chappelle, and Y. R. Chen. 2001. Multispectral steady-state fluorescence imaging system for plant leaves. Appl. Optics 40:157-166.

Lang, M., P. Stiffel, Z. Braunova, and H. K. Lichtenthaler. 1992. Investigation of the blue-green fluorescence emission of plant leaves. Bot. Acta 105: 395-468.

Lu, R., and Y. R. Chen. 1998. Hyperspectral imaging for safety inspection of food and agricultural products. in pathogen detection and remediation for safe eating. SPIE 3544: 121-133.

Martinsen, P., P. Schaare, and M. Andrews. 1999. A versatile near infrared imaging spectrometer. J. Near Infrared Spectrosc. 7: $17-25$.

Mehl, P. M., Y. R. Chen, and M. S. Kim. 2000. Development of detection methods for defects and contaminations on apple surface using hyperspectral imaging technique. Opt. Eng. [Submitted for publication].

Miller, W. M., J. A. Throop, and B. L. Upchurch. 1998. Pattern recognition models for spectral reflectance evaluation of apple blemishes. Postharvest Biol. Tech. 14(1): 11-20.

Papageorgiou, G. 1975. Chlorophyll fluorescence: An intrinsic probe of photosynthesis. In Bioenergetic of Photosynthesis, 320?371. Govindjee, ed. New York, N.Y.: Academic Press.

Park, B., Y. R. Chen, and M. Nguyen. 1998. Multi-spectral image analysis using neural network algorithm for inspection of poultry carcasses. J. Agri. Engr. Res. 69: 351-363.

Pen, C. L., W. K. Bilanski, and D. R. Fuzzen. 1987. Classification analysis of good and bruised peeled apple tissue using optical reflectance. Trans. ASAE 28(1): 326-330.

Rehkugler, G.E., and J. A. Throop. 1986. Apple sorting with machine vision. Trans. ASAE 29(5): 1388-1397.

Smillie, R. M., S. E. Hetherington, R. Nott, G. R. Chaplin, and N. L. Wade. 1987. Application of chlorophyll fluorescence to the postharvest physiology and storage of mango and banana fruit and the chilling tolerance of mango cultivars. ASEAN Food J. 3 : $55-59$.

Tao, Y., and Z. Wen. 1999. An adaptive spherical image transform for high-speed fruit defect detection. Trans. ASAE 42(1): 241-246.

Upchurch, B. L., H. A. Affeldt, W. R. Hruschka, K. H. Norris, and J. A. Throop. 1990. Spectrophotometric study of bruises on whole, 'Red Delicious' apples. Trans. ASAE 33(2): 585-589. 